\title{
Das Unrecht des Bürgers
}

Jochen Bung*

\section{Rezension zu Michael Pawlik: Das Unrecht des Bürgers. Grundlinien der Allgemeinen Verbrechens- lehre, Tübingen (Mohr Siebeck) 2012, 510 S., 114 €, ISBN 978-3-16-152189-8}

Das von Michael Pawlik vorgelegte, in acht Jahren Arbeit entstandene beachtliche Buch verfolgt ein ehrgeiziges Ziel. Es möchte dem Strafrecht eine systematische Einheit und Geschlossenheit vermitteln, von der zweifelhaft ist, ob es sie überhaupt jemals hatte oder haben kann. Die Systemansprüche werden in zum Teil harschen Verdikten artikuliert. Insbesondere Dualismen werden als Anzeichen dafür genommen, dass etwas mit dem Denken nicht stimmt (vgl. S. 85 f. Fn. 448, S. 239 f. Fn. 519, s. auch S. 215 Fn. 399). Selbst Hegel, neben Jakobs wichtigster Gewährsmann von Pawlik, bleibt nicht unbeanstandet, ansonsten aber ist die Hegelsche Logik und die Hegelsche Rechtsphilosophie die maßgebliche Inspirationsquelle von Pawliks Systemdenken.

\section{Mitwirkung, Zuständigkeit und Zurechnung}

Das Unternehmen betrifft freilich zunächst einmal nur den Allgemeinen Teil oder mit Pawliks Worten - die „Allgemeine Verbrechenslehre“. Der Besondere Teil des Strafrechts partizipiert nicht an der Dignität, aus der normativen Infrastruktur von Begriffen entwickelt werden zu können (vgl. S. 45 f.). Der Allgemeine Teil aber, so Pawliks methodisches Verständnis, erschließt sich aus begrifflichen Interdependenzen, die der Rekonstruktion eines Ableitungszusammenhangs zugänglich sind. Dessen konzeptuelle Grundmarkierungen sind (wofür auch die drei Kapitel des Buches stehen): Mitwirkung, Zuständigkeit und Zurechnung: Strafrechtliche Zurechnung ergibt sich aus einem System der Zuständigkeiten, und dieses System ist die Folge des normativen Mechanismus staatsbürgerlicher Mitwirkung in der Bewährung von gleichursprünglichen Rechten und Pflichten. Im Gedanken der Mitwirkungspflicht tut sich allerdings ein großes Spektrum auf, vom bloßen Gehorsam bei Hobbes bis zur indirekten oder direkten Partizipation bei Locke und Rousseau. Pawlik kritisiert unbarmherzig die Idee, dass legitimes Strafrecht sich aus dem Gedanken herschreiben muss, dass die Adressaten zugleich auch die Autoren jener Normen sind, die ihnen Verhalten unter Androhung von Strafe vorschreiben oder verbieten. Gegenüber solchen „gerechtigkeitstheoretischen Exaltationen“ (S. 108 Fn. 594), beharrt Pawlik auf der gleichsam primordialen Bedeutung des Staates und seinem Anspruch, ein Leben in Frieden und Sicherheit zu ermöglichen. Mitwirkung ist also wesentlich Gehorsam und erstreckt sich unter anderem auf die Pflicht, bei der Aufklärung von Straftaten mitzuwirken, und zwar (insoweit wieder unhobbesianisch) unabhängig 
davon, ob man gerade Beschuldigter ist oder nicht (S. 107 Fn. 592). Die Funktionalisierung von Freiheit für die Sicherheit scheint ein wesentliches Anliegen von Pawliks „freiheitsfunktionalem“ Verständnis der Rechtsgesellschaft zu sein.

Wenig ergiebig für die Illumination des begrifflichen Zusammenhangs von Mitwirkung, Zuständigkeit und Zurechnung ist eine aufgewärmte Strafzweckdiskussion, die nichts Neues zu Tage fördert und nur die alten Melodien repetiert (S. 52 ff., S. 61 ff., S. 82 ff.). Pawliks Buch belegt, was niemand klarer sah als Nietzsche: Wenn man zu wahren Einsichten im Strafrecht kommen will, muss man die Strafzweckdiskussion hinter sich lassen. Die maßgeblichen Argumente Pawliks für die Herausarbeitung seines Ableitungszusammenhangs hängen, auch wenn die Darstellung anderes glauben machen möchte, nicht entscheidend von Strafzweckgesichtspunkten ab. Sie werden auch im Laufe des Buches nicht wieder aufgegriffen, was ihre argumentative Entbehrlichkeit erweist. „Festhalten“ an der Mitverantwortung durch Strafe, Verdeutlichung des Zusammenhangs von Freiheitsgenuss und Mitwirkungspflicht (vgl. S. 110) ist allen Strafzwecken gegenüber offen und nichtssagend. Die Grundidee Pawliks ist vielmehr eine Variation von Kants Bestimmung des Begriffs vom Recht. Was den Rechtszustand charakterisiert, ist die sozialverträgliche (nicht nur bestandserhaltende, sondern auch entwicklungsermöglichende) Abstimmung von Freiheitssphären. Die Aufgabe dieser Abstimmung erledigt nicht primär das Recht selbst, sondern es übernimmt gleichsam die Schirmherrschaft über das Gesellschaftsspiel, in dem die Inhaber dieser Sphären unter Beweis stellen, dass ihnen die Abstimmung gelingt. Dieses Gelingen ist nämlich der wesentliche Inhalt der Mitwirkungspflicht, die sich aus den Spielregeln des Gesellschaftsvertrags ergibt. Sie begründet eine basale Garantenstellung derart, dass die Bürgerinnen und Bürger für die sozialverträgliche Moderation ihrer Grundfreiheiten zuständig sind. Die Generalpflicht der Bürgerinnen und Bürgers ist, sozusagen, für praktische Konkordanz zu sorgen (vgl. zum Begriff eines wechselseitigen Sich-in-die-Schranken-Verweisens, S. 238, zum Anspruch des anderen als „immanente Schranke meiner Rechtssphäre“, S. 144).

Aus diesem Gedanken entwickelt Pawlik am Vorbild der Hegelschen Vorstellung von der Vermittlung des Allgemeinen und Besonderen bzw. der „,bestimmten Allgemeinheit" (S.150) eine umfassende, nicht mehr nur auf den Bereich der sog. unechten Unterlassungsdelikte beschränkte Systematik der Garantenpflichten (vgl. S. 159 ff.), die vollständig natürlich nur unter Einschluss des Gesichtspunkts positiver Freiheit ist (Respektierungszuständigkeiten $u n d$ Ermöglichungszuständigkeiten, vgl. S. 177), was zwar nicht unmittelbar in Kants Rechtsbegriff enthalten, vermittelt über den kategorischen Imperativ, mit dem bekanntlich auch Hilfspflichten begründet werden, dann aber doch auch konstitutives Merkmal des kantianischen Rechtszustands ist. Die rechtliche Zurechnung einer Pflichtverletzung lässt sich schließlich aus der 
normativen Anforderung deduzieren, dass das Tun und Unterlassen der Rechtsperson darauf gerichtet ist, sich selbst unter größtmöglicher Schonung der legitimen Interessen anderer zur Geltung zu bringen - ein Gedanke, den Pawlik im Begriff der Bemühensobliegenheit (vgl. S. 310 f., S. 334 f., S. 341 ff.) sublimiert.

\section{Vorsatz, Fahrlässigkeit und Verbotsirrtum}

Zurechnung folgt also aus Zuständigkeiten. Das Zurechnungssystem selbst lässt sich gleichfalls als begrifflicher Zusammenhang rekonstruieren. Hier ist die Grundidee folgende: Vorsatz ist rekonstruierbar als Fahrlässigkeit und Fahrlässigkeit mit einem generalisierten Verständnis des vermeidbaren Verbotsirrtums vermittelbar (der Täter ignoriert eine Pflicht). Mit Nachdruck verwirft Pawlik die herrschende Aliud-Konzeption des Verhältnisses von Vorsatz und Fahrlässigkeit als „typologisches“ Denken, dem er unbestechlich strenge Systematik entgegensetzen will (vgl. S. 364 o.): Fahrlässigkeit ist Zuständigkeitspflichtverletzung - dass jemand sich zu sehr auf Kosten der legitimen Interessen anderer verwirklicht hat. Vorsatz bedeutet dann qualifizierte Zuständigkeitspflichtverletzung (S. 344 Fn. 528, S. 373) Das ist begrifflich nicht falsch, aber epiphänomenal, ohne relevanten Informationsgehalt, weil die Zuständigkeitspflichtverletzung im Falle der Vorsatztat schließlich nicht der Grund des Handelns war.

Zustimmung verdient Pawlik aber für seine Kritik an der „axiologischen Unstimmigkeit“ (S. 395) des geltenden Rechts in Form der $\mathbb{S} \mathbb{1 6}, 17$ StGB. Betrachten wir zwei klassische Fälle: Der Täter hat keine Rechtsauskunft eingeholt, bevor er seine Tätigkeit aufgenommen hat. Der Täter hat nicht genau hingesehen, als er mit dem Jagdgewehr Schüsse abgegeben hat. Nicht genau hinzuschauen ist nicht durchweg weniger verzeihlich, als keinen Expertenrat einzuholen, so dass die Möglichkeit, den Verbotsirrtum als Vorsatztat abzuurteilen gegenüber der Generalmilderung des $\mathbb{1 6}$ I 2 StGB ungerecht erscheint. Andererseits findet - und das wäre wohl als systematischer Kern der Kontroverse zu verorten - nach gut begründbarer Auffassung die krimino-axiologische Stimmigkeit in einer Regel wie Art. 21 S. 2 des schweizerischen Strafgesetzbuchs Ausdruck, wonach der vermeidbare Verbotsirrtum stets zu Strafmilderung führt: Obligatorische statt fakultative Strafmilderung. Pawlik hält die fakultative Lösung für vorzugswürdig (vgl. S. 406 Rn. 880). Es existierten nämlich Fälle hartnäckiger Missachtung der Garantenobliegenheiten, die es rechtfertigten, den Täter genauso streng zu bestrafen wie den Vorsatztäter. Pawlik selbst betont aber, dass dies seltene Fälle sein müssen („seltene Ausnahme“, S. 408 Fn. 894). Ich vermute sogar, dass es Fälle sind, in denen sich der (vermeintliche) Unachtsamkeitsfall von dem einer Vorsatztat überhaupt nicht mehr unterscheiden lässt. Wenn jemand beschließt: „Ich will jetzt ganz bewusst nicht genau hinsehen“ und in der Folge dieser Weigerung einen Menschen und kein Wildschwein erschießt, dann ist die Möglichkeit, dass das Zielobjekt auch ein Wildschwein hätte sein können für die 
strafrechtliche Zurechnung irrelevant: Inkaufnehmen und damit Im-RechtssinneBilligen des Unrechtserfolgs. Man kommt hier überhaupt nicht zur Frage eines Irrtums und daher glaube ich auch, dass Pawliks Einwand, meiner Konzeption des Grenzbereichs von Vorsatz und Fahrlässigkeit mangele es an einem „belastbaren tertium komparationis“ (S. 395 Fn. 808), kein schlagender Einwand ist. Etwas ist Vorsatz oder Fahrlässigkeit, tertium non datur. Es führt zu Scheinkontroversen, wenn Pawlik den Willensbegriff strategisch re-psychologisiert, um dann ein Gegenargument gegen jene zur Verfügung zu haben, denen er einen zu wenig objektivierten oder normativierten Vorsatzbegriff vorhält.

Warum ist beim vermeidbaren Verbotsirrtum die obligatorische Milderung statt der fakultativen richtig? Der Grund liegt darin, dass die axiologisch und phänomenologisch stimmige Konzeption des Verhältnisses von Vorsatz und Fahrlässigkeit die eines Aliud-Verhältnisses ist. Mit dieser Konzeption ist verträglich, dass man aufgrund von Fahrlässigkeit Vorsatz hat (freilich aufgrund des von Pawlik abgelehnten zweistufigen Zurechnungssystems, vgl. S. 263 f.). Was dann aber vorzuwerfen ist, ist Fahrlässigkeit und nicht Vorsatz (in typologischer Sprache: „Schwerpunkt der Vorwerfbarkeit“). Der Verbotsirrtum, wie er in $\$ 17$ geregelt ist, leistet folgender Lesart Vorschub: Wer fahrlässig vorsätzlich handelt, handelt vorsätzlich und nicht fahrlässig. Dagegen muss es heißen: Wer fahrlässig vorsätzlich handelt, handelt fahrlässig und nicht vorsätzlich. Es gibt keinen vorsätzlichen Irrtum. Und auch die Normativierung und Objektivierung des Subjektiven hat eine Grenze. Das sieht Puppe ganz richtig, der Pawlik aber Inkonsequenz vorhält (vgl. S. 392 ff.). Die normative Objektivation des Wollens kommt da an eine Grenze, wo ich möglicherweise nicht weiß, was ich will, aber mir die Gründe fehlen, es wissen zu wollen. Niemals geht es freilich um strikt individuelles Wollen - individuum ineffabile est. Aber gerade deswegen, weil es, jedenfalls in einem modernen und aufgeklärten Strafrecht, um die Ermittlung einer gerechten Mitte zwischen Individuum und Maßstabsperson geht, ist es sinnvoll, mit der herrschenden Fahrlässigkeitsprüfung eine objektive und eine subjektive Sorgfaltspflichtverletzung bei objektiver und subjektiver Voraussehbarkeit zu differenzieren (vgl. S. 261 f., Fn. 32). In seiner Kritik an diesem Ansatz unterläuft Pawlik eine petitio principii: Dass die Prüfung der subjektiven Zurechnung nicht zweistufig, sondern einstufig zu erfolgen hat (S. 335 Fn. 776), folgt bereits aus dem Begriff der subjektiven Zurechnung und besagt nichts gegen den Sinn einer zweistufigen Prüfung.

Die Diskussion kann hier natürlich nicht erschöpfend geführt werden, deswegen will ich hier noch einmal die Eckpunkte benennen: Zustimmung zur Kritik an der axiologischen Unstimmigkeit der unterschiedlichen Behandlung von Tatumstands- und Verbotsirrtum, Zustimmung im Übrigen auch zur Kritik an der (prominent von Radbruch geäußerten) Aliud-Auffassung des Verhältnisses von Begehungs- und Unterlassungdelikten (vgl. S. 161). Keine Zustimmung aber an der Kritik der Aliud- 
Auffassung des Verhältnisses von Vorsatz und Fahrlässigkeit. Wenn wir diese - meinetwegen „typologische“ - Unterscheidung kassieren, geht m.E. die Vorstellung darüber verloren, was das Unrecht des Bürgers zum Kriminalunrecht - zum besonders schlimmen Unrecht - macht. Zwischen den Zeilen von Pawliks elegant klingender freiheitsfunktionaler Zuständigkeitslehre schlummert ein gigantisches freiheitsvernichtendes Kriminalisierungsprogramm. Er bleibt eine Auskunft darüber schuldig, wieweit die „Bereitschaft zur Aufmerksamkeit“ (S. 308) Teil strafrechtlicher Verantwortungszuschreibung werden soll. „Eine freiheitsfunktional konzipierte strafrechtliche Zurechnungslehre muss somit die Forderung an die Bürger: „Unterlasst zuständigkeitswidrige Handlungen! “ um eine Erwartung zweiter Ordnung ergänzen: „Sorgt im Rahmen des Zumutbaren dafür, dass ihr dieser Forderung mit hinreichender Verlässlichkeit nachzukommen vermögt!“(S. 310) Aber es bleibt unklar, in welchem Umfang diese Erwartungen zweiter Ordnung strafrechtlich flankiert werden müssen. Als Hinweise finden sich der Gedanke der Sozialadäquanz (S. 336), ein unklarer Gedanke der „Wirklichkeitsmächtigkeit“ der Verhaltensnorm (S. 325) sowie die subjektiven Kriterien der Gleichgültigkeit und Rücksichtslosigkeit (S. 397). Nicht widersprechen wird man dem Autor sicherlich, wenn er äußert, „die strafrechtlich verlangte Sorgfalt [sei] auf ein solches Maß zu beschränken, das sich realistischerweise dauerhaft durchhalten lässt“ (S. 342).

\section{Vom Unrecht zum Kriminalunrecht des Bürgers}

Kriminalunrecht, so die einfache Idee, die ich dagegen setzen würde, besteht zwar nicht ausschließlich, aber dem Grunde nach nur da, wo man den Täter fragen kann: Warum hast du das getan? Wie in Bertolt Brechts Gedicht über den minderjährigen Elternmörder Jakob Apfelböck ist eine mögliche und häufige Antwort auf diese Frage: Ich weiß es nicht. Vielleicht ist das wahr, vielleicht auch nicht, vielleicht ist der Täter verrückt. Das Strafrecht weiß es in vielen Fällen besser und antwortet: Weil du es wolltest. Stellt sich heraus, dass der Täter verrückt ist, weil er Menschen für Dämonen gehalten hat, ändert es nichts an seinem Wollen, aber an seinem Dafürkönnen, denn der Täter wusste nicht, was er wollte: Er wollte Menschen töten, aber er wusste es nicht. $\$ 17 \mathrm{StGB}$ ist aber gegenüber $\mathbb{1 6} \mathrm{StGB}$ auch in den Fällen nichtverrückten Handelns stets die bessere Regelung, weil sie dem Umstand Rechnung trägt, dass der Bedeutungsgehalt des Wollens maßgeblich durch Faktoren außerhalb des Wollenden bestimmt wird und die Aufgabe des Strafrechts in der Tat darin besteht, dem Täter die Deutungshoheit über die Bedeutung seiner Handlungen zu entziehen.

Die Frage, ob jemand etwas dafür kann, dass er nicht wusste, was er wollte, ist die Frage der Vermeidbarkeit. Wenn man nicht in einen kriteriellen Regress geraten will, muss man aus der Begründung aussteigen und entscheiden. Am Grunde aller Entscheidungen liegt eine Wahrnehmung von etwas als vorsätzlich oder fahrlässig. Wer 
dieses normative Wahrnehmungsurteil kassieren(oder „vermitteln“) möchte, erzeugt am Ende nicht mehr Systematik, sondern ein System, an dem die Anwender irre werden.

Pawliks Darstellung markiert nicht den Punkt, an dem das „Unrecht des Bürgers“ zum Kriminalunrecht wird. Die Unvollständigkeit seines Ableitungszusammenhangs lässt sich an mehreren Stellen greifen, z.B. heißt es auf S. 257: „Zurechenbar-zuständigkeitswidrig [...] verhält sich, wer die ihm aus seiner Zuständigkeit erwachsende Verhaltenspflicht unerfüllt lässt, obgleich er sie hätte erfüllen können, wenn er jenes $\mathrm{Maß}$ an Bemühungen an den Tag gelegt hätte, welches die Rechtsgemeinschaft von ihren Mitgliedern erwartet. Dies ist dann der Fall, wenn der Täter zurechnungsfähig und es ihm zumutbar war, den Inhalt der Verhaltenspflicht zu erkennen und ihr nachzukommen. Eine Zurechnungsunterbrechung lässt sich deshalb abgesehen von dem Fall der Zurechnungsunfähigkeit nur in jenen verhältnismäßig seltenen Konstellationen legitimieren, in denen die inneren oder äußeren Hemmungsfaktoren pflichtgemäßen Verhaltens derart schwer wiegen, dass der Umstand, ihnen erlegen zu sein, die Rechtstreue des Täters nicht in Zweifel zieht. Liegt keiner dieser Fälle vor, so steht fest, dass der Betreffende Kriminalunrecht verwirklicht hat. " Leider ist in der Argumentation die Prämisse nicht enthalten, welche die Aufnahme des Ausdrucks „Kriminal“ in der Schlussfolgerung tragen würde.

Ebenso an anderen Stellen: „Allgemein gesprochen, bleibt der Straftäter hinter den Anforderungen der für einen Bürger in seiner Situation maßgeblichen Verhaltensnorm zurück, weil er sich nicht in dem rechtlich erwarteten Ausmaß um ein pflichtgemäßes Verhalten bemüht hat. Dieses tatsächlich manifestierte Nein des Täters, der von ihm an den Tag gelegte Mangel an Rechtstreue erlaubt es, ihm sein Verhalten als Mitwirkungspflichtverletzung und damit als kriminelles Unrecht zuzurechnen.“ (S. 298) Erneut bleibt unklar, was die Schlussfolgerung rechtfertigen könnte. Pawliks „und damit“ ist ein non sequitur. Die an anderen Stellen sich findenden allgemeinen Aufgabenbeschreibungen des Strafrechts, „einen Zustand konkret-realer Freiheitlichkeit aufrechtzuerhalten“ (S. 329) sind viel zu allgemein, um das Spezifikum und die Ratio des Strafrechts hervortreten zu lassen. Alle Rechtsgebiete arbeiten (jedenfalls in einem Rechtsstaat) daran, einen Zustand konkret-realer Freiheitlichkeit aufrechtzuerhalten. Was ist das Besondere des Strafrechts?

Zweifellos kann sich Pawlik darauf zurückziehen, dass sein Modell mit jeder Kriminalisierung kompatibel ist, die ein legitimer Gesetzgeber zu verantworten hat. Gleichwohl ist die Bestimmung: „Kriminelles Unrecht zu begehen heißt [...]: die Verpflichtung zu verletzen, an der Aufrechterhaltung eines Zustands der Freiheitlichkeit mitzuwirken" (S. 258) in dieser Form unzureichend. 


\section{Fazit und offene Fragen}

Selbst wenn ich keiner einzigen These des Buches zustimmen würde (was nicht der Fall ist), müsste ich feststellen, dass sich die deutsche Strafrechtswissenschaft froh schätzen darf, dass Bücher dieser Art noch aus ihrem Zusammenhang heraus entstehen. Pawliks Untersuchung enthält eine nahezu unüberschaubare Fülle an Diskussionsanregungen und sie zwingt dazu, sämtliche Grundbegriffe des Allgemeinen Teils des Strafrechts auf den Prüfstand zu stellen. Die Gelehrtheit des Autors ist auf jeder Seite zu spüren, man lernt eine ganze Menge, insbesondere über Dogmengeschichte. Vielleicht, aber das ist keine inhaltliche Kritik, hätte dem Werk zuweilen etwas mehr Leichtigkeit der Formulierung („Mauern der hiesigen Konzeption“, S. 407 u. dgl.) und etwas Distanz zu den großen Referenztexten gut getan. Die HegelRezeption ist zu unvermittelt, bei der Diskursivierung eines so schwierigen Autors wie Hegel müssen mehr Übersetzungsleistungen erbracht werden, als es Pawlik tut, der sich häufig nur auf den Wortlaut des Meisterdenkers verlässt.

Nicht frei von Kritik ist auch die zu beobachtende Fetischisierung des Systemdenkens. Zuweilen wirkt es, als wolle der Autor mehr Systematik um der puren Systematik willen. Dass es aber keine widerspruchsfreien Systeme gibt, wissen wir nicht erst seit Gödel. Das Leben selbst ist voller Widersprüche. Im Leben und in der Bewältigung des Lebens um jeden Preis systematische Einheit herstellen zu wollen, führt in die Irre oder zu Zwangsvorstellungen. Gegen diesen Systemwahnsinn, konkret: die Iteration von Sorgfaltsanforderungen, muss das unmittelbare Eigenrecht der Phänomene zur Geltung gebracht werden. Sie lassen sich nicht auf Begriffe und Begriffsverhältnisse reduzieren. In modernen Gesellschaften ist ein „axiologisch einheitliches Profil des Bürgers" schwerlich möglich (S. 295).

Systeme sind aber nicht nur notwendigerweise widersprüchlich, sie trennen häufig auch Dinge, die zusammengehören. Dies (die von Pawlik ironisierte „BauklötzchenDenkweise, vgl. S. 152) ist aber der Preis für die Operationalisierbarkeit der Systembegriffe, wie sie etwa auch in dem - sicherlich mit Mängeln behafteten - herkömmlichen Straftataufbau Ausdruck finden. Die Nagelprobe des besseren Systems wird sein, ob der Aufbau nach Tatbestandsmäßigkeit, Rechtswidrigkeit und Schuld aufgegeben wird. Ich prognostiziere, dass dies nicht der Fall sein wird. Es ist eben, wie auch Pawlik konzediert, „sachgerecht [...], auf eine etwaige vorrangige Zuständigkeit des Verletzten erst einzugehen, nachdem die (anhand des einschlägigen Deliktstatbestandes konkretisierte) Ausgangszuständigkeit des Täters ermittelt worden ist." (S. 218). Pawlik liefert auch den Grund, aus dem deutlich wird, dass man ohne diese Prüfungsfolge, überhaupt erst gar nicht zur Prüfung käme: „Der Grund dafür liegt in dem Umstand, dass es dessen Strafbarkeit [d.i. die des Täters, J.B.] ist, die in Rede steht [...]“ (S. 218). 
Michael Pawlik hat zweifellos eines der spannendsten strafrechtlichen Bücher der letzten Jahre vorgelegt. Durch das Bisherige wurden - wenn auch notwendigerweise unvollständig - bereits zentrale Schauplätze der Diskussion deutlich. Aber das Buch enthält noch viele andere. Sie seien hier abschließend, ohne Anspruch auf Vollständigkeit, kursorisch angesprochen:

1. Pawlik meint, intrinsische Gerechtigkeitsansprüche des Rechts ließen sich nicht sprechakttheoretisch begründen, das führe in einen Zirkel (vgl. S. 40 f.). Das halte ich für unzutreffend. Es ist kein Zirkel, deontologische Gehalte des Rechts auf sprachvermittelte protokontraktuale Anerkennungsverhältnisse zu gründen. „Man muss [...] bereits eine Haltung der Anerkennung eingenommen haben“ (S. 41), sagt Pawlik, und genau diese Haltung hat man eingenommen, wenn man sich mit anderen verständigt. Die „Ermöglichungsbedingungen [...] rechtlichen Anerkanntseins“ (S. 186) liegen, wie bereits Hobbes wusste, in der Sprache, deren institutioneller Ausdruck der Sprechakt ist.

2. Wieso ist Resozialisierung lediglich ein „Reflex einer gesellschaftspolitisch nützlichen Strategie“ (S. 74)? Diese Behauptung müsste zumindest im Lichte der bundesverfassungsgerichtlichen Rechtsprechung überprüft werden, wonach sich der Resozialisierungsanspruch des Gefangenen aus der Menschenwürde ergibt.

3. Pawlik gegen Gierhake (S. 126 Fn. 694): Gierhake begründe „zwar die Staatsunabhängigkeit der Strafe als eines reinen Gedankendings, nicht aber auch jene der tatsächlich vollstreckten Strafe“. Das mag daran liegen, dass die tatsächliche Vollstreckung völkerrechtlicher Strafen einstweilen in der Hand der Staaten liegt (vgl. 106 IStGH-Statut). Hier liegt tatsächlich ein Legitimationsproblem, über das weiter nachgedacht werden muss.

4. Der Rechtsgutsbegriff ist in seiner kritischen Potenz überschätzt, da hat Pawlik völlig Recht (vgl. S. 127 ff.). Als technischer Ausdruck zur Klassifikation von Delikten hat er sich bewährt. Darüber hinaus ist er ziemlich uninteressant.

5. Zur den strafrechtlichen Figuren der eigenverantwortlichen Selbstverletzung (S. 219 ff.) und der Notwehr (S. 237 ff.) finden sich wichtige Anknüpfungspunkte, unter anderem auch eine begrifflich schärfere Begründung der bekannten sozialethischen Einschränkung des Notwehrrechts. Hingewiesen sei in diesem Zusammenhang auf die wichtige und dogmatisch ausbaufähige Figur der „prinzipiellen Legalitätsvermutung" (S. 247).

6. Pawlik kritisiert den Tu-quoque-Ansatz bei der Begründung der Unzumutbarkeit normgemäßen Verhaltens (vgl. S. 350). Sein eigener Erklärungsversuch - „[d]as jetzige Opfer hätte sich ebensogut in der Rolle des Notstandstäters wiederfinden können und umgekehrt“ (S. 361) sieht einem Tu-quoque-Argument ziemlich ähnlich. 
7. Pawliks Ablehnung des Zwei-Stufen-Systems der Zurechnung, das auf der Unterscheidung von Handlungs- und Beweggründen beruht, überzeugt mich nicht: „Weshalb soll an dieser Unterscheidung festgehalten werden?“ (S. 264) Gegenfrage: Warum nicht? Die Unterscheidung von Handlungen, Handlungsgründen und Motiven ist so elementar in unsere Verständigungspraxis über Fragen der Verantwortung eingelassen, dass jeder Gegenentwurf die Begründungslast trägt. Dass es „[k]onstruktiv denkbar wäre“ (S. 264), die beiden Stufen einzuebnen, heißt noch nicht, dass es sinnvoll ist, einer solchen Entdifferenzierung Vorschub zu leisten. Pawlik meint: „Auf der Basis einer im Personell-Geistigen kulminierenden Anthropologie setzt sinnhaftes Handeln Schuldhaftigkeit voraus. “ (S. 275) Das mag sein, begründet aber nicht, dass Strafrecht auf der Basis einer im Personell-Geistigen kulminierenden Anthropologie entwickelt werden sollte. Die von Pawlik kritisierte „technizistische Engführung “ (S. 294) des instrumentellen Handlungsbegriffs betrifft nur die erste Stufe der Zurechnung und ermöglicht ein methodisch kontrolliertes, nämlich schrittweises Einbeziehen weiterer Persönlichkeitsanteile, weshalb er allen Gesamtbetrachtungsmodellen eindeutig vorzuziehen ist. 